\title{
250 SPATIAL-TRANSCRIPTOMIC ANALYSIS OF TUMOR- IMMUNE MICROENVIRONMENT IN AML PATIENTS RECEIVING PEMBROLIZUMAB AND DECITABINE
}

${ }^{1}$ Chen Zhao*, ${ }^{1}$ Abigail Wong-Rolle, ${ }^{2}$ Prajan Divakar, ${ }^{3}$ Katherine Calvo, ${ }^{4}$ Christopher Hourigan. 'National Cancer Institute, North Bethesda, MD, USA; ${ }^{2}$ NanoString, Seattle, WA, USA; ${ }^{3}$ National Institutes of Health Clinical Center, Bethesda, MD, USA; ${ }^{4}$ National Heart, Lung, and Blood Institute, Bethesda, MD, USA

Background Relapsed or refractory Acute Myeloid Leukemia (R-AML) is a deadly disease with an inadequate response rate to current treatments. Recent advances in immunotherapy shed light on R-AML, and several clinical trials have shown promising potential for combining immune checkpoint inhibitors (ICIs) with hypomethylating agents. A deeper understanding of the tumor-immune microenvironment in R-AML during combination ICI treatment is urgently needed for developing better therapeutics and stratifying treatment strategies.

Methods To dissect the tumor-immune interactions in the bone marrow microenvironment, we employed nanoString GeoMx Digital Spatial Profiler (DSP) and performed a spatialtranscriptomic analysis of patients with R-AML who received pembrolizumab and decitabine. We compared the transcriptomic profiles and TCR clonalities of tumor-interacting T cells, bystander $\mathrm{T}$ cells, and other cells at baseline, post-pembrolizumab treatment, and post-decitabine, which enable us to identify R-AML's suppressive immune microenvironment and immune cells' responses to ICI and hypomethylating agent.

Results We obtained the spatial-transcriptomic profiles of $\mathrm{T}$ cells, stromal cells, and leukemia cells in patients with R-AML at different treatment points. Our TCR-specific probes were able to track $\mathrm{T}$ cell clonal changes during treatments.

Conclusions R-AML harbored a complex tumor immune microenvironment and diverse $\mathrm{T}$ cell clonality.

Acknowledgements This research was supported in part by the Intramural Research Program of the NCI (the Center for Cancer Research), NHLBI, and NIH Clinical Center.

Ethics Approval This study is approved by NHLBI IRB.

http://dx.doi.org/10.1136/jitc-2021-SITC2021.250 